seems to have been so warm that instead of choosing between alternative payloads for a single satellite, two have been adopted for the early 1970s. The second, provisionally called UK 5, breaks new ground. For the first time, American experiments will be included in a British payload and carried in a British-built spacecraft. The satellite will be concerned with cosmic X-rays, and experiments from the University of Leicester group, University College, London, and possibly Imperial College, are in view. In the new field of X-ray astronomy, British scientists have gained an enviable position through an experiment on the first Anglo-American satellite and also from stabilized Skylark rocket flights from Woomera. So far, American experimenters have had limited opportunities for this kind of work. The Astronomy, Space and Radio Board is very happy about the inclusion of the American experiments, which it hopes will help repay American hospitality. UK 5 will be a relatively sophisticated vehicle, and may take some years to complete. A design study by an industrial firm is at present being arranged. The other new satellite, UK 4, can go ahead faster, as it makes use of spare flight equipment prepared for last year's Aeriel 3, and will carry similar radio experiments.

\section{Radio Telescope in Space}

RADIO astronomers in Britain must this week bo envious of their Amcrican colleagues who, if all goes well, will soon have at their disposal a satellite designed purely with radio astronomy in mind, and equipped to pick up low frequency radio signals from space which are not able to penetrate the ionosphere. The striking feature of the new satellite, the Radio Astronomy Explorer-A (RAE-A), is its two V-shaped aerials; the open end of one $V$ pointing downward at the Earth, the other $\mathrm{V}$ directed outwards. When the two aerials are fully deployed, the $\mathrm{X}$-shaped configuration which the structure will then have will be $1,500 \mathrm{ft}$ from tip to tip, or as long as the Empire State building is tall.

The interest of RAE-A, launched on July 4, is that it will be able to extend radio astronomy measurements to parts of the radio spectrum inaccessible from the Earth. So far, attempts to carry out this kind of measurement from satellites have been relatively crude. Ariel 2, for instance, carried an experiment to detect cosmic radio noise between $750 \mathrm{kHz}$ and $3 \mathrm{MHz}$ picked up by a small dipole antenna, a type of antenna which has poor directional qualities. RAE-A is the next step after the early elementary experiments; when fully deployed its enormous $V$ antennae will have a beam width, in the plane of the aerial, of about $30^{\circ}$ when it is receiving signals at a frequency of $3 \mathrm{MHz}$, compared with a beamwidth of $120^{\circ}$ for a dipole.

The deployment of such large aerials in space is not without its difficulties, however, and previous attempts to extend much shorter aerials from spinning spacecraft have been known simply to wrap the aerials around the body of the vehicle. The Americans are therefore being cautious on this occasion. The plan is to stop the spin of RAE-A once it has been injected into its final orbit at an altitude of 3,730 miles by releasing weights on the ends of wires, eliminating any small remaining spin by lining up the spacecraft in the Earth's magnetic field. Once this is done, the aerial booms can be extended, at first only to a length of $450 \mathrm{ft}$. They will remain at this length, gathering data, for a fortnight, while their behaviour is monitored by four TV cameras, which also serve to determine the source of the radio signals picked up by the aerials. If all goes well, the booms will then be extended a further $300 \mathrm{ft}$ to their maximum length.

Linear acrials such as the acrials on RAE-A just now seem the most promising configurations for satellite radio astronomy, as the dish aerials used for groundbased work are too heavy to be carried into space, even if they are made of metallic foil. This is one of the reasons radio astronomers are watching with interest the deployment of the $750 \mathrm{ft}$ booms, which mark quite an advance in space technology. At launch, the booms are stored within RAE-A as spools of flat tape, of silver-plated beryllium-copper alloy, $5 \mathrm{~cm}$ wide and $0.005 \mathrm{~cm}$ thick. As a motor unreels the spool, the tape curls to form a rigid tube about $1.25 \mathrm{~cm}$ in diameter. One of the problems is expected to be distortion of the tubes by the heating effect of sunlight to avoid this the tape is perforated to allow sunlight to penetrate the tube and heat it more uniformly.

The two aerials are not the only booms making RAE-A look more like a spider than a spacecraft. It also has two $60 \mathrm{ft}$ booms forming a dipole acrial, and a $630 \mathrm{ft}$ boom which is to help damp out small oscillations of the satellite.

In its 3,730 mile orbit, well above the blanketing effect of the ionosphere and most of the Earth's halo of electrons, RAE-A should do a good job. Its receivers are sensitive to nine frequencies in the range $450 \mathrm{kHz}$ to $9 \cdot 2 \mathrm{MHz} ; 10 \mathrm{MHz}$ is roughly the frequency below which signals cannot penetrate the ionosphere. The aerial pointing toward space will be used to map the galaxy at low frequencies. Low frequency measurements of radiation which may come from cosmic ray particles should help in understanding the origin of the particles and how they are accelerated, as well as giving some information on the magnetic fields in space. The upper aerial will also collect low frequency measurements of the Sun, a means of studying the corona out to distances about a third of the way to the Earth. The V-shaped aerial pointing downwards to the Earth will, on the other hand, be able to investigate radio emission from near the Earth, and the $120 \mathrm{ft}$ long dipole is to follow the radio bursts from Jupiter. The configuration of the two $V$ aerials, one pointing toward space and the other Earthwards, should help in separating cosmic signals and signals coming from the Earth's environment, hitherto one of the problems of radio astronomy satellites.

\section{Report from Wales}

A NEW variety of spring oat which is resistant to three races of mildew was granted plant breeders rights in December 1967. Called Mostyn, it has bcen developed at the Welsh Plant Breeding Station, Aberystwyth, whose annual report for 1967 (price 10s.) includes a description of the new variety. Mostyn has been developed from a complex cross involving the wild oat (Avena sterilis) which contributed the resistance to the mildew fungus, and three other varieties, Powys, Pennant and Condor. Mostyn, which combines high grain yields with resistance to mildew, appeared 